\title{
RESPONSABILIDAD SOCIAL Y SOSTENIBILIDAD EN LAS BIBLIOTECAS UNIVERSITARIAS ESPAÑOLAS
}

\author{
José-Luis Herrera-Morillas, Ana Castillo-Díaz y Margarita Pérez-Pulido
}

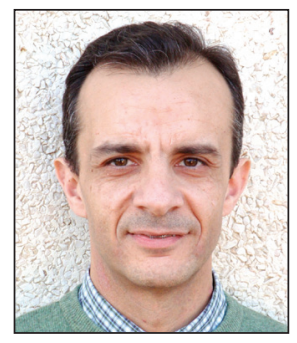

José-Luis Herrera-Morillas es doctor en documentación por la Universidad de Granada, donde cursó también la licenciatura en Historia del Arte y la diplomatura en Biblioteconomía y Documentación. En la actualidad es profesor titular en la Facultad de Ciencias de la Documentación y la Comunicación de la Universidad de Extremadura, en la que imparte diferentes materias como organización de colecciones; información y sociedad; o fondo antiguo. Ha publicado varios libros y artículos especializados sobre estos temas.

http://orcid.org/0000-0001-5310-643X

jlhermor@alcazaba.unex.es

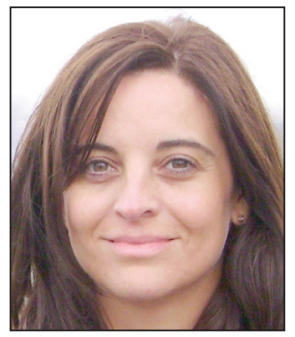

Ana Castillo-Díaz es doctora en comunicación por la Universidad de Extremadura. Licenciada en ciencias de la información, especialidad de publicidad por la Universidad de Sevilla. Es profesora contratada en la Facultad de Ciencias de la Documentación y la Comunicación de la Universidad de Extremadura, en la que imparte diferentes materias como comunicación audiovisual y publicitaria digital; comunicación corporativa e interna o teoría de la comunicación y de la información. Entre sus líneas de investigación están la comunicación institucional, comunicación empresarial e institucional online y la comunicación y salud. Es co-autora de los libros Imagen y comunicación en las pymes (2005), Comunicación integral y responsabilidad social en las organizaciones" (2009). http://orcid.org/0000-0002-3650-2964

acasdia@alcazaba.unex.es

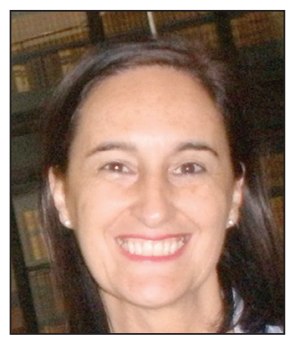

Margarita Pérez-Pulido es doctora en documentación por la Universidad de Salamanca, donde cursó también la licenciatura en historia del arte y la diplomatura en biblioteconomía y documentación. Es profesora titular en la Facultad de Ciencias de la Documentación y la Comunicación de la Universidad de Extremadura, en la que imparte diferentes materias como planificación, auditoría y Evaluación de Unidades de información; información y sociedad; o ética y deontología de la información. Ha publicado varios libros y artículos especializados y ha participado en congresos nacionales e internacionales sobre estos temas. Es miembro del grupo de ética de Sedic y pertenece a la Oficina de Responsabilidad Social de la Universidad de Extremadura.

http://orcid.org/0000-0003-2831-6341

marga@alcazaba.unex.es

Universidad de Extremadura Facultad de Ciencias de la Documentación y la Comunicación Plazuela de Ibn Marwan, s/n. 06071 Badajoz, España

\section{Resumen}

Se recopilan y analizan las iniciativas y recursos relacionados con la responsabilidad social y sostenibilidad en las bibliotecas universitarias españolas. La información se ha obtenido de los sitios webs de las bibliotecas y de otros documentos elaborados por ellas: memorias de actividades, folletos, planes estratégicos y documentos sobre gestión de la colección. Para organizar y estudiar la información se ha elaborado una lista de análisis formada por 5 categorías que abarcan un total de 21 elementos. 61 de las 71 bibliotecas universitarias analizadas $(85,9 \%)$, incluyen alguna actividad relacionada con la responsabilidad social y sostenibilidad.

\section{Palabras clave}

Responsabilidad social, Sostenibilidad, Biblioteca verde, Bibliotecas universitarias, España.

Title: Social responsibility and sustainability in the Spanish university libraries 


\begin{abstract}
This compilation and analysis of the initiatives related to social responsibility and sustainability in 71 Spanish university libraries obtained information primarily from web sites and print materials: activities reports, brochures, strategic plans and collection management documents. To organise and analyse the information, a list of 5 categories was developed, covering 21 elements. In 61 (85.9\%) of the universities analysed, some type of library activity related to social responsibility and sustainability was identified.
\end{abstract}

\title{
Keywords
}

Social responsibility, Sustainability, Green library, University libraries, Spain.

Herrera-Morillas, José-Luis; Castillo-Díaz, Ana; Pérez-Pulido, Margarita (2014). "Responsabilidad social y sostenibilidad en las bibliotecas universitarias españolas". El profesional de la información, marzo-abril, v. 23, n. 2, pp. $134-143$.

http://dx.doi.org/10.3145/epi.2014.mar.05

\section{Introducción}

En la sociedad actual crece la inquietud por las cuestiones ecológicas y sociales. "Existe una preocupación creciente por problemas tales como el deterioro medioambiental imputable a las actividades económicas, las prácticas laborales discriminatorias, la violación de los derechos humanos, el subdesarrollo, etc." (Nieto-Antolín; Fernández-Gago, 2004). Desde el punto de vista de las organizaciones, Zadek (2007) opina que han ido evolucionando desde una etapa defensiva hasta adquirir compromisos que promueven una participación amplia en la responsabilidad social (etapa civil).

La responsabilidad social (RS) es un término que hace referencia a los compromisos legales y éticos derivados de los impactos que la actividad de las organizaciones provoca en el sector social, laboral, medioambiental y de los derechos humanos. En este contexto las organizaciones se preocupan cada vez en mayor medida por obtener la legitimación social dando muestras evidentes de su implicación en cuestiones relacionadas con la $\mathrm{RS}$.

Para las universidades esa necesidad de legitimación es mucho más acuciante por su marcada función social. El concepto de responsabilidad social universitaria (RSU) define "una nueva manera de funcionamiento de la universidad, un nuevo modo de hacer y comportarse basado en una relación más fluida y directa de la universidad con su entorno social, para lo que se toman en consideración los efectos, repercusiones y expectativas que la actividad de la universidad genera tanto en sus propios miembros (docentes, investigadores, personal de administración y estudiantes) como en la sociedad" (Pérez-Domínguez, 2009).

Cada vez hay más iniciativas de RSU, por ejemplo destacamos en América Latina el caso del sistema educativo chileno, en donde la RSU se desarrolla a través del proyecto Universidad: construye país (Fernández; Delpiano; De-Ferrari, 2006, citado por Martínez-Pichardo; Hernández-Oliva, 2013), o también, las prácticas llevadas a cabo por Ausial (asociación de las 31 universidades confiadas a los jesuitas en 15 países de América Latina) (Gargantini; Zaffaroni, 2011).

Navarro-García (2012) habla de "RS aplicada" al referirse a la existencia de una gran variedad de términos para ajustarse al tipo de organización responsable. De este modo:
- responsabilidad social corporativa (RSC) es la aplicable a las empresas;

- responsabilidad social organizacional (RSO) es más amplia e incluye la RSC propia de las empresas y además la de todo tipo de organizaciones no lucrativas y la administración pública;

- responsabilidad social universitaria (RSU) se aplica a la universidad;

- responsabilidad social corporativa interna (RSCI), es la de la empresa con los trabajadores, propia de la gestión de recursos humanos;

- responsabilidad social individual (RSI), es un término de nuevo cuño por el que respondemos por nuestros actos como consumidor, trabajador, directivo o ciudadano.

\section{Las organizaciones se preocupan por ob-} tener legitimación social dando muestras de su implicación en cuestiones relacionadas con la responsabilidad social

Independientemente de la aplicación a un contexto determinado este autor atribuye unos conceptos esenciales a la RS:

- responsabilidad (la globalización es un fenómeno irreversible);

- legitimidad social (conocida y aceptada por la sociedad y la comunidad internacional);

- toma de decisiones prudente, libertad (las organizaciones son un elemento indispensable para el desarrollo de la sociedad);

- voluntariedad (la RS se mueve en el ámbito de la ética).

Con el objetivo de unificar y establecer criterios de uso, en 2005 ISO creó un Grupo de Trabajo sobre RS cuyo resultado ha sido años después la norma ISO 26000:2010, convertida en marzo de 2012 en UNE-ISO 26000:2012. En ella aparecen definiciones, principios, orientaciones, directrices sobre la aplicación de RS en gobernanza de la organización, derechos humanos, prácticas laborales, medio ambiente, prácticas justas de operación, asuntos de consumidores, participación activa y desarrollo de la comunidad (Aenor; Garrigues, 2012). 
Sin embargo, no todas las acciones de RS tienen que traducirse necesariamente en buena reputación para las organizaciones. Refiriéndose a las empresas, Villafañe (2004) sostiene que "la acción filantrópica entendida como una suerte de peaje social, que es otra forma de entender la RSC por algunas empresas, no perjudica a la reputación corporativa pero tampoco la beneficia".

En el caso de las universidades, algunos autores creen conveniente sustituir el concepto RSU por el de sostenibilidad universitaria (SU) al considerar la RS un componente filantrópico e instrumento de construcción de imagen, por lo que se aconseja superar la filantropía y la extensión universitaria por un compromiso trasversal, progresivo y multidireccional (Marraud, 2013).

Sostenible es introducir la ética de la información en los programas educacionales y elaborar códigos de ética con valores (Nolin, 2010)

Nolin (2010) relaciona los conceptos de desarrollo sostenible e información. Define sostenibilidad como el conjunto de estrategias de protección ambiental, igualdad social y desarrollo económico en la idea de que es posible combinar el crecimiento económico con estos tres pilares que la sostienen. Se basa en las distintas actuaciones de las Naciones Unidas desde la Comisión Mundial de Medio Ambiente y Desarrollo en 1987, 1992 y 1996, donde se añade a los tres pilares el desarrollo cultural, y en 2002 cuando anuncia la llamada Década de la educación para el desarrollo sostenible, 2005-2014. La relación entre desarrollo sostenible e información la encontramos, según este autor, en el desarrollo de tecnología de la información sostenible, el uso eficiente de estas tecnologías, las prácticas y funciones de la producción del conocimiento, el establecimiento de indicadores y normas para la toma de decisiones, la igualdad de acceso a la información, y el almacenamiento y conservación sostenible de la información, en diferentes culturas, contextos e instituciones. Sostenible es igualmente introducir la ética de la información en los programas educacionales y elaborar códigos de ética con valores.

Podemos hablar de conceptos propios de sostenibilidad y RS como estrategia corporativa y como algo trasversal incrustado en la cultura corporativa de una organización.

El objetivo de este trabajo es trazar un cuadro general de las iniciativas y recursos relacionados con la RS y sostenibilidad en las bibliotecas universitarias españolas. De este modo se pretende dar a conocer qué bibliotecas están trabajando en este ámbito y con qué planteamientos y actuaciones. También se persigue que los datos aportados sirvan de estímulo para las bibliotecas que quieran iniciar o mejorar sus implicaciones en este asunto.

\section{Responsabilidad social y biblioteca}

En el contexto internacional hay que destacar el Environmental Sustainability and Libraries creado como grupo de interés especial de la IFLA. En 2002, con ocasión de su 75 aniversario, se aprueba en Glasgow el Statement on libraries and sustainable development, que dice que el desarrollo sostenible es una preocupación esencial de las bibliotecas en sus aspectos más concretos de igualdad de acceso a la información, multiculturalidad, educación, libertad intelectual y valores democráticos en general.

Otra iniciativa está relacionada con el concepto de green library, con origen en la sección Biblioteca y medio ambiente del Boletín de la Biblioteca Wilson (Antonelli, 2008). Para obtener información al día se puede consultar el sitio Green libraries, dedicado a la información sobre la biblioteca verde y la sostenibilidad, sobre todo en Norteamérica.

http://www.greenlibraries.org

En mayo de 2011 tuvo lugar en Marília (Brasil) el IX Encuentro de la Asociación de Educación e Investigación en Ciencia de la Información de Iberoamérica y el Caribe (Edicic 2011) bajo la denominación Investigación y enseñanza en las ciencias de la información: ética y responsabilidad social, en el que se presentan varias comunicaciones que abordan la RS en relación con los profesionales y los servicios de información (Múnera-Torres, 2011; Rodrigues-Bernardino, 2011; Núñez, 2011; Gracioso et al., 2011; Caldas; Carlos, 2011). http://www.edicic.org/ix_edicic.html

En España podemos citar algunas iniciativas de profesionales de bibliotecas en RS en los últimos años. En primer lugar, la presentación del Foro Sectorial de Cooperación para el Desarrollo y Bibliotecas durante las IX Jornadas españolas de documentación de 2009.

\section{Existe concienciación sobre la importan- cia de la gestión de la responsabilidad social y sostenibilidad en casi la mitad de las bibliotecas universitarias españolas}

En 2010 se celebró la III Jornada profesional de la Red de Bibliotecas del Instituto Cervantes (RBIC) bajo la denominación Desarrollo, sostenibilidad y cultura: el papel social de las bibliotecas, con el propósito de dar a conocer "las fórmulas y herramientas que las bibliotecas han puesto en marcha en cooperación al desarrollo, sostenibilidad, derechos humanos, multiculturalidad, etc."

http://www.cervantes.es/bibliotecas documentacion espanol/para_bibliotecarios/jornadas/jornada_3/ jornada_3.htm

Organizadas por Sedic, en 2011 se Ilevaron a cabo las XIII Jornadas de gestión de la información, con el título De la responsabilidad al compromiso social dedicadas a los temas de identidad y memoria, derechos humanos, atención a grupos desfavorecidos y colaboración con otros agentes, papel de las TIC en las movilizaciones sociales y ética de la información. Esta misma institución organizó, también en 2011, la jornada profesional La responsabilidad social corporativa en las organizaciones, en las que se presentaron trabajos de interés como el de Gallego-Rubio y Gimeno-Perelló (2011) dedicado a la RSC como estrategia de la Biblioteca de la Universidad Complutense. 
http://www.sedic.es/xiiijornadas_gestinfo-convocatoria.asp http://www.sedic.es/actividades-jornada_RSC_30marzo2011.asp

Las bibliotecas que más destacan por sus aportaciones a la responsabilidad social y sostenibilidad son las de Cádiz, Burgos, Huelva y Carlos III de Madrid

En el contexto de las bibliotecas universitarias las actuaciones en materia de RS vienen marcadas por la política universitaria española y las acciones de Rebiun. La Ley orgánica de universidades trata de la necesidad de prestar servicios a la comunidad con las características propias de RS. El estudio de la Comisión técnica de la Estrategia universidad 2015 denominado La responsabilidad social de la universidad y el desarrollo sostenible, incorpora una tercera misión de la universidad (además de la docencia y la investigación), la "transferencia del conocimiento y tecnología y RSU" y comienzan a crearse oficinas de RS en varias universidades.

El III Plan estratégico Rebiun 2020 incorpora en una de sus líneas el desarrollo de la RS de la universidad. El informe Contribución de las bibliotecas en materia de RS y sostenibilidad universitarias, propone la adopción de los criterios de RS y sostenibilidad por parte de la propia organización, y la integración de las bibliotecas universitarias en las estrategias, planes y políticas de RS de sus instituciones. El informe busca un enfoque práctico en la incorporación de prácticas de RS en las bibliotecas universitarias españolas, a varios niveles: a) en la integración de la biblioteca en las iniciativas de su institución adaptándose a su contexto (certificación de sistemas de gestión ambiental, prácticas y políticas laborales, acción social y cooperación para el desarrollo, código deontológico, participación en comités y grupos de trabajo); b) en aspectos propios de la profesión (edificios, colecciones, procesos, servicios, programas y planes estratégicos, formación de usuarios); y c) en la propia actitud del profesional (compromiso moral con el desarrollo tecnológico y la evolución económica social de hoy). En su parte más teórica, el informe trata de la caracterización del concepto RSU y su sustitución por el de sostenibilidad universitaria.

\section{Metodología}

Una de las primeras tareas de este trabajo ha consistido en localizar y recopilar información sobre las iniciativas y recursos relacionados con la RS y sostenibilidad de las bibliotecas universitarias, para seguidamente clasificarlas de un modo organizado que facilite su posterior análisis.
Se ha tenido en cuenta el citado informe de Rebiun (2012), en concreto, los cuatro apartados que establece para ubicar las contribuciones e iniciativas en esta materia:

- Edificios bibliotecarios

- Colecciones

- Procesos y servicios bibliotecarios

- Formación de usuarios

A partir de la explicación de estos apartados y de la bibliografía consultada se ha elaborado un modelo basado en los apartados de Rebiun, pero incluyendo algunos elementos nuevos que se han considerado significativos al estar presentes en las bibliotecas analizadas, y omitiendo otros al no aparecer en las mismas. El modelo ha servido de ayuda para localizar y, sobre todo, clasificar la información recopilada.

La información se ha obtenido de los sitios web de las bibliotecas y de otros documentos elaborados por ellas: memorias de actividades, folletos, planes estratégicos, y documentos sobre gestión de la colección. Es decir, los soportes que las bibliotecas utilizan para fijar sus estrategias y difundirlas.

El modelo o lista para el análisis consta de 5 categorías que abarcan 21 elementos (tabla 1):

- Programas o declaraciones de principios sobre RS. Recoge las bibliotecas que presentan documentos dedicados a la
Tabla 1: Distribución del número de bibliotecas por categorías

\begin{tabular}{|c|c|}
\hline Categorías & $N^{\circ}$ de bibliotecas \\
\hline C1-Programas o declaraciones de principios sobre RS y sostenibilidad & 7 \\
\hline 1. Descripción & 7 \\
\hline 2. Logotipo & 5 \\
\hline 3. Comités, grupos de trabajo & 4 \\
\hline C2-Planes estratégicos & 30 \\
\hline 4. Líneas, ejes & 18 \\
\hline 5. Objetivos & 14 \\
\hline 6. Otros & 15 \\
\hline C3-Edificios & 8 \\
\hline 7. Ahorro energético & 5 \\
\hline 8. Adaptación para discapacitados & 5 \\
\hline 9. Residuos y reciclaje & 7 \\
\hline 10. Panel de difusión & 2 \\
\hline C4-Colecciones & 60 \\
\hline 11. Consorcios y licencias & 36 \\
\hline 12. Open access & 17 \\
\hline 13. Repositorios & 41 \\
\hline 14. Adquisiciones & 14 \\
\hline 15. Política de expurgo, duplicados & 17 \\
\hline 16. Control del consumo & 6 \\
\hline 17. Fomento de la lectura & 11 \\
\hline 18. Redes colaboración & 4 \\
\hline C5-Procesos y servicios bibliotecarios & 20 \\
\hline 19. Servicios y préstamos especiales & 16 \\
\hline 20. Cursos, campañas o actividades sobre RS & 2 \\
\hline 21. Medidas de concienciación & 6 \\
\hline
\end{tabular}


descripción de los principios, medidas, compromisos o actividades relacionadas con la RS. También se precisa si han diseñado un logotipo al respecto y si cuentan con comités o grupos de trabajo (grupo de trabajo biblioteca verde, comisión deontológica).

- Planes estratégicos de las bibliotecas. Indica si hay menciones en estos documentos relacionadas con la RS, fundamentalmente dentro de las líneas o ejes estratégicos, en los objetivos y también en otros apartados como valores, compromisos o visión. Estos documentos se diferencian de los de la categoría anterior en que son generales de planificación bibliotecaria, mientras que los anteriores plasman exclusivamente la postura sobre la RS.

- Edificios bibliotecarios. Abarca las actuaciones sobre las medidas de ahorro energético, adaptación para discapacitados (mobiliario y equipamiento para discapacidades de movilidad y visual), eliminación de residuos (separación, puntos limpios, etc.) o los paneles de difusión para informar de estos temas. Es decir, medidas concretas relacionadas con la RS y los edificios.

- Colecciones. Refleja las actuaciones que fomentan el ahorro, como la participación en consorcios (no se tiene en cuenta la pertenencia a Rebiun), la práctica y promoción del open access, creación y mantenimiento de repositorios, política de adquisiciones que cuente con una reglamentación de su gestión económica y presupuestaria (dentro de los planes o normas de gestión de la colección, favoreciendo de este modo centrarse en lo que se necesita), política de expurgo y duplicados, medidas limitadoras para el control del consumo de papel y tóner; e iniciativas de colaboración y solidaridad como el fomento de la lectura o la participación en redes de colaboración que promueven el compromiso humanitario, la cooperación al desarrollo y las donaciones.

- Procesos y servicios bibliotecarios. Categoría destinada a plasmar: los servicios y préstamos especiales (para usuarios con discapacidad física, hospitalizados); los cursos, campañas o actividades de carácter monográfico sobre las implicaciones de la RS de las bibliotecas; las medidas de concienciación, como la elaboración de guías, folletos, documentos de buenas prácticas..., o la inclusión de aspectos sobre el uso responsable y sostenible de la biblioteca en los cursos que se imparten en las bibliotecas.

El conjunto de universidades españolas se ha obtenido del documento Listado alfabético de universidades por comunidades autónomas.

http://www.ua.es/es/internet/listado.htm

La información de los sitios web se ha obtenido mediante consultas realizadas en agosto y septiembre de 2013.

\section{Resultados}

De las 71 bibliotecas españolas analizadas, 61 (85,9\%) incluyen algún tipo de aportación relacionada con la RS y sostenibilidad.

Los recursos e iniciativas más extendidos (tabla 1) están relacionados con las categorías:

- Colecciones: 60 bibliotecas (98,3\%)

- Planes estratégicos: 30 (49,1\%)
- Procesos y servicios bibliotecarios: 20 (32,7\%)

- Edificios bibliotecarios: 8 (13,1\%)

- Programa o declaración de principios: 7 (11,4\%)

Las bibliotecas que más destacan por sus aportaciones a la RS son las de Cádiz, Burgos, Huelva y Carlos III de Madrid (tabla 2). Todas ellas coinciden en gestionarla de una manera organizada y cuentan hasta con un logotipo.

Aunque la responsabilidad social y sostenibilidad es una cuestión presente en las bibliotecas universitarias españolas, en buen número de ellas aparece de manera dispersa y poco visible

Como ejemplo ilustrativo de las bibliotecas analizadas se puede citar la de Cádiz y sus iniciativas, entre las que destacan:

- Creación del Comité de responsabilidad social para "identificar las áreas que en esta materia presentan posibilidades de mejora, proponiendo alternativas localizadas a través de benchmarking, entrevistas con expertos...".

- Elaboración de código deontológico.

- Diseño de logotipo.

- Menciones en su plan estratégico, por ejemplo el objetivo "desarrollar políticas de conciliación, salud laboral e impacto medioambiental" que se concreta en varias medidas; o el valor "contribuir al desarrollo y defensa del medio ambiental potenciando las políticas medioambientales fomentadas por la Universidad".

- Colocación de contenedores para la separación de residuos en todas sus instalaciones.

Tabla 2: Bibliotecas con más aportaciones sobre RS

\begin{tabular}{|l|c|}
\hline \multicolumn{1}{|c|}{ Bibliotecas universitarias } & No de aportaciones \\
\hline Cádiz & 17 \\
\hline Burgos & 13 \\
\hline Huelva & 11 \\
\hline Carlos Ill de Madrid & 11 \\
\hline Jaén & 10 \\
\hline Complutense de Madrid & 9 \\
\hline Politècnica de Catalunya & 8 \\
\hline Oviedo & 8 \\
\hline Málaga & 7 \\
\hline Sevilla & 7 \\
\hline Uned & 7 \\
\hline Murcia & 7 \\
\hline Politécnica de Cartagena & 7 \\
\hline Granada & 6 \\
\hline Pablo Olavide & 6 \\
\hline Autònoma de Barcelona & 6 \\
\hline Barcelona & 6 \\
\hline Extremadura & 6 \\
\hline La Rioja & 6 \\
\hline
\end{tabular}




\begin{tabular}{|c|c|c|c|c|}
\hline Burgos & Cádiz & Carlos III & Huelva & Oviedo \\
\hline $\begin{array}{l}\text { Biblioteca } \\
\text { Verde }\end{array}$ & Biblioteca $)$ (Sostenible & & liotecc & \\
\hline
\end{tabular}

Logotipos

- Campaña Escribe en verde destinada a la reutilización del papel. Colocación de bandejas con papel a disposición de los alumnos en las salas de lectura.

- Medidas de adaptación de mobiliario y equipamiento para discapacidades de movilidad y visual, y para los alumnos del aula de mayores (curso 2009-10).

- Instalación en todas las sedes de un panel con el lema Biblioteca responsable, para colgar información relativa a RS.

- Servicio de préstamo para personas con discapacidad.

- Fomento del ahorro de papel con mensajes alusivos en el pie de los correos.

- Supresión de impresión de las "hojas de tránsito" en el procedimiento del préstamo.

- Elaboración de una guía abreviada sobre los tipos y niveles de discapacidad con que se puede encontrar el personal de la biblioteca.

\section{Conclusiones}

Existe concienciación sobre la importancia de la gestión de la RS y sostenibilidad en casi la mitad de las bibliotecas universitarias españolas, y así lo plasman de maneras dispares en los planes estratégicos por medio de líneas estratégicas, objetivos o en apartados donde desarrollan sus valores, visiones o compromisos corporativos.

Una de las variables más significativas para indicar que las bibliotecas ya están llevando a cabo una gestión de la RS consciente, intencionada y elaborada es la de Programas o declaraciones de principios, que se concreta en aspectos como la creación de un logotipo específico o un comité de trabajo. Sin embargo, la cifra de esta variable implica afirmar que la puesta en práctica de programas de gestión de la RS en las bibliotecas universitarias españolas es todavía escasa e incipiente.

La variable Edificios bibliotecarios, que mide actuaciones como las medidas de ahorro energético o eliminación de residuos, también está muy vinculada a la gestión consciente de la RS y al igual que sucede con la variable Programas o declaraciones de principios, alcanza un valor reducido y sirve para corroborar la anterior afirmación.

La pequeña presencia de Cursos, campañas o actividades, y Medidas de concienciación también se puede utilizar para apoyar la necesidad de potenciar más la gestión de la RS en las bibliotecas objeto de estudio y mejorar la formación en este ámbito.

El apartado Colecciones es el que destaca en mayor medida. Las nuevas tendencias de gestión de la colección contribuyen al ahorro y a la sostenibilidad y por ello contribuyen a su vez de manera consciente o no a fomentar la RS en la organización. La clave estaría en dilucidar si las bibliotecas que fomentan estas actuaciones son o no conscientes de que están apoyando la RS. Los datos anteriores pueden llevar a afirmar que existe una preocupación por el ahorro, pero que no siempre se plasma en un planteamiento formal y riguroso sobre la gestión de la RS.

Hay que señalar que las actuaciones de fomento de la lectura, con un gran componente de compromiso social, tradicionalmente han sido más propias de otros tipo de bibliotecas como las públicas o las escolares; el hecho de que ahora estén presentes en las bibliotecas universitarias refleja un

Tabla 3. Etapas en el desarrollo de la responsabilidad social corporativa

\begin{tabular}{|l|l|l|}
\hline \multicolumn{1}{|c|}{ Etapa } & \multicolumn{1}{|c|}{ Qué hacen las organizaciones } & \multicolumn{1}{c|}{ Por qué lo hacen } \\
\hline Defensiva & Niegan prácticas, efectos o responsabilidades. & $\begin{array}{l}\text { Para defenderse contra ataques a su reputación que podrían en el corto } \\
\text { plazo afectar las ventas, el reclutamiento, la productividad y la marca. }\end{array}$ \\
\hline Cumplimiento & $\begin{array}{l}\text { Adoptan un enfoque de cumplimiento basado en } \\
\text { políticas, como un costo de hacer negocios. }\end{array}$ & $\begin{array}{l}\text { Para mitigar la erosión de valor económico en el mediano plazo debido a } \\
\text { riesgos continuos de reputación y litigios. }\end{array}$ \\
\hline Gestión & $\begin{array}{l}\text { Introducen el tema social dentro de sus procesos } \\
\text { centrales de gestión. }\end{array}$ & $\begin{array}{l}\text { Para mitigar la erosión de valor económico en el medio plazo y obtener } \\
\text { ganancias de más largo plazo al integrar prácticas de negocios responsa- } \\
\text { bles en sus operaciones diarias. }\end{array}$ \\
\hline Civil & $\begin{array}{l}\text { Integran el tema social en sus estrategias centrales } \\
\text { de negocios. }\end{array}$ & $\begin{array}{l}\text { Para mejorar el valor económico a largo plazo y obtener ventajas al ser los } \\
\text { primeros en alinear la estrategias y las innovaciones con el tema social. }\end{array}$ \\
\hline
\end{tabular}


mayor vínculo hacia el compromiso social. Lo mismo sucede, pero ya dentro de la variable Procesos y servicios bibliotecarios con la puesta en marcha de servicios y préstamos especiales para personas con discapacidad.

Tras esta revisión de la información analizada es posible concluir que aunque la RS y sostenibilidad es una cuestión que está presente en las bibliotecas universitarias españolas, en buen número de ellas aparece de manera dispersa y poco visible. Puede que incluso no sean conscientes de que la practican. Por ello se hace evidente una falta de gestión de la comunicación de todas las cuestiones relacionadas con la RS que ya realizan y una metodología de puesta en práctica. Una propuesta de mejora es que estas bibliotecas identifiquen y hagan más visibles la contribución que ya hacen a la RS, para luego completarla con otras actuaciones como las que presentamos en este trabajo.

Por otra parte, queda pendiente de analizar la identificación de las bibliotecas con la política de RSU de sus instituciones, ya que de acuerdo con los documentos analizados en la parte inicial de este trabajo es una cuestión importante la necesidad de identificarse con ellas y colaborar en todo aquello que tengan como objetivo las oficinas de RSU. Ésta es en principio una cuestión más compleja que no queda totalmente reflejada en las fuentes utilizadas y requiere un trabajo de investigación cualitativa más completo que se abordará en futuras investigaciones.

\section{Bibliografía}

Aenor; Garrigues, Abogados y Asesores Tributarios (2012). Principios, prácticas y beneficios de la responsabilidad social. Madrid: Aenor Ediciones. ISBN: 9788481437553

Antonelli, Monika (2008). "The green library movement: an overview and beyond". Electronic green journal, v. 1, n. 27. http://escholarship.org/uc/item/39d3v236

Berbes-Cardós, José-Antonio (2002). "Servicios de sala y préstamo en el contexto actual". En: Magán-Wals, José-Antonio (coord.). Temas de biblioteconomía universitaria y general, pp. 440-459. Madrid: Editorial Complutense. ISBN: 8474916674

Bernardino, Maria-Cleide-Rodrigues (2011). "Responsabilidade social e aplicação da ciência da informação: amparada nos discursos dos teóricos". Revista Edicic, v. 1, n. 2, pp. 71-81. http://www.edicic.org/revista/index.php?journal=RevistaE DICIC\&page $=$ article\&op=view\&path []$=27$

Caldas, Rosângela-Formentini; Carlos, Gisnai-Da-Silva (2011). "O enfoque da ciência da informação no contributo ao desenvolvimento sustentável regional: o caso do arquivo da ACIM". Revista Edicic, v. 1, n. 2, pp. 212-226.

http://www.edicic.org/revista/index.php?journal=RevistaE DICIC\&page $=$ article \&op=view \&path []$=36$

Comisión de las Comunidades Europeas (2001). Libro verde: fomentar un marco europeo para la responsabilidad social de las empresas.

http://eur-lex.europa.eu/LexUriServ/site/es/com/2001/ com2001_0366es01.pdf

Comisión Técnica de la Estrategia Universitaria 2015 (2011). La responsabilidad social de la universidad y el desarrollo sostenible. Madrid: Secretaría General de Universidades. ISBN: 9788436952278

http://goo.gl/PzJdNe

Gracioso, Luciana-De-Souza; Zafalon, Zaira-Regina; Boccato, Vera-Regina-Casari; Gaspar, Nádea-Regina; Ferraz, Maria-Cristina-Comunian; Hoffmann, Wanda-AparecidaMachado; De-Faria, Leandro-Innocentini-Lopes (2011). "Reformular para flexibilizar: ampliação da formação profissional em prol da responsabilidade social". Revista Edicic, v. 1, n. 2, pp. 35-52.

http://www.edicic.org/revista/index.php?journal=RevistaE DICIC\&page $=$ article\&op=view $\&$ path []$=25$

Fernández, Carola; Delpiano, Catalina; De-Ferrari, JoséManuel (edits.) (2006). Responsabilidad social universitaria. Una manera de ser universidad. Teoría y práctica en la experiencia chilena. Santiago de Chile: Universidad construye país. ISBN: 9568140085

http://rsuniversitaria.org/web/images/stories/memoria/ UCP\%202006.pdf

Gallego-Rubio, Cristina; Gimeno-Perelló, Javier (2011). “La responsabilidad social corporativa: una visión estratégica de la Biblioteca de la Universidad Complutense de Madrid". En: Jornada profesional La responsabilidad social corporativa en las organizaciones, Madrid, 30 marzo 2011.

http://www.sedic.es/actividades-jornada_RSC_30marzo2011.asp

Gargantini, Daniela; Zaffaroni, Cecilia (coords.) (2011). Informe final del proceso de autoevaluación de la responsabilidad social universitaria en Ausjal. Córdoba (Argentina): Alejandría Editorial. ISBN: 9789871780013

http://goo.gl/Yhypm4

IFLA (2002). Statement on libraries and sustainable development. http://www.ifla.org/III/eb/sust-dev02.html

Magán-Wals, José-Antonio; Gimeno-Perelló, Javier (2008). “¿Es 'bibliotecariamente correcto' el compromiso social de la biblioteca universitaria?". Educación y biblioteca, v. 20, n. 166, pp. 95-101.

http://gredos.usal.es/jspui/handle/10366/119558

Marraud, Gerardo (2013). Las bibliotecas y la responsabilidad social universitaria: informe de Rebiun.

http://eprints.rclis.org/20107/1/RSC\%20Rebiun_ Fesabid\%202013.pdf

Martínez-Pichardo, Pascacio-José; Hernández-Oliva, Alejandrina-Victoria (2013). "Responsabilidad social universitaria: un desafío de la universidad pública mexicana". Contribuciones desde Coatepec, v. 12, n. 24, enero-junio, pp. 85-103. http://www.redalyc.org/pdf/281/28126456001.pdf

Múnera-Torres, María-Teresa (2011). "La responsabilidad social del profesional de la ciencia de la información". Revista Edicic, v. 1, n. 1, pp. 135-147. http://goo.gl/8FKOAK

Navarro-García, Fernando (2012). Responsabilidad social corporativa: teoría y práctica. 2a ed. rev. y aum. Madrid: ESIC. ISBN: 9788473568241

Nieto-Antolín, Mariano; Fernández-Gago, Roberto (2004). "Responsabilidad social corporativa: la última innovación 
en management". Universia business review - Actualidad económica, primer trimestre, pp. 28-39.

http://ulegid.unileon.es/admin/UploadFolder/63_(2004).pdf

Nolin, Jan (2010). "Sustainable information and information science". Information research, v. 15, n. 2, june http://informationr.net/ir/15-2/paper431.html

Núñez, Mónica (2011). “Desafíos de las ciencias de la información y su responsabilidad social ante los nuevos escenarios societales: notas para la discusión". Revista Edicic, v. 1, n. 1, pp. 168-182.

http://www.edicic.org/revista/index.php?journal=RevistaE DICIC\&page $=$ article\&op=view $\&$ path []$=16$

Pérez-Domínguez, Fernando (2009). La responsabilidad social universitaria (RSU). Huelva: Consejo Social de la Univer- sidad de Huelva.

http://rsuniversitaria.org/web/images/stories/Memoria\%20 RSU\%20Huelva\%202009.pdf

Rebiun (2012). Contribución de las bibliotecas en materia de responsabilidad social y sostenibilidad universitarias.

http://goo.gl/Z5t22M

Rebiun. III Plan estratégico de REBIUN 2020.

http://www.mcu.es/bibliotecas/docs/MC/ConsejoCb/CTC/ Bib_Univ/Planestrategico2020.pdf

Villafañe, Justo (2004). La buena reputación. Claves del valor intangible de las empresas. Madrid: Pirámide. ISBN: 8436818393

Zadek, Simon (2005) "El camino hacia la responsabilidad corporativa". Hardvard business review, agosto, pp. 3-10. http://rse-global.org/autoresponder/rse_el_camino_zadek.pdf

\section{Anexo 1. Lista de evaluación}

\begin{tabular}{|c|c|c|c|c|c|c|c|c|c|c|c|c|c|c|c|c|c|c|c|c|c|c|c|c|}
\hline C1-Programas... & A & B & C & D & $\mathbf{E}$ & $\mathbf{F}$ & G & $\mathbf{H}$ & $\mathbf{I}$ & $\mathbf{J}$ & $\mathbf{K}$ & $\mathbf{L}$ & $M$ & $\mathbf{N}$ & $\tilde{\mathbf{N}}$ & 0 & $\mathbf{P}$ & $\mathbf{Q}$ & $\mathbf{R}$ & S & $\mathbf{T}$ & $\mathbf{U}$ & $\mathbf{v}$ & w \\
\hline Descripción & 0 & 1 & 0 & 0 & 1 & 1 & 0 & 0 & 0 & 0 & 0 & 0 & 0 & 0 & 0 & 1 & 0 & 0 & 0 & 0 & 0 & 0 & 0 & 0 \\
\hline Logotipo & 0 & 1 & 0 & 0 & 1 & 0 & 0 & 0 & 0 & 0 & 0 & 0 & 0 & 0 & 0 & 1 & 0 & 0 & 0 & 0 & 0 & 0 & 0 & 0 \\
\hline Comités, grupos & 0 & 1 & 0 & 0 & 1 & 1 & 0 & 0 & 0 & 0 & 0 & 0 & 0 & 0 & 0 & 1 & 0 & 0 & 0 & 0 & 0 & 0 & 0 & 0 \\
\hline C1-Programas... & $x$ & $\mathbf{Y}$ & $\mathbf{z}$ & AA & BB & $\mathrm{CC}$ & DD & $\mathrm{EE}$ & $\mathbf{F F}$ & GG & HH & II & JJ & KK & LL & MM & NN & $\tilde{\mathbf{N} N \tilde{N}}$ & 00 & PP & $\mathbf{Q Q}$ & $\mathbf{R R}$ & SS & TT \\
\hline Descripción & 0 & 0 & 0 & 0 & 0 & 0 & 0 & 0 & 0 & 0 & 0 & 0 & 0 & 0 & 0 & 0 & 1 & 0 & 0 & 0 & 0 & 0 & 0 & 0 \\
\hline Logotipo & 0 & 0 & 0 & 0 & 0 & 0 & 0 & 0 & 0 & 0 & 0 & 0 & 0 & 0 & 0 & 0 & 1 & 0 & 0 & 0 & 0 & 0 & 0 & 0 \\
\hline Comités, grupos & 0 & 0 & 0 & 0 & 0 & 0 & 0 & 0 & 0 & 0 & 0 & 0 & 0 & 0 & 0 & 0 & 0 & 0 & 0 & 0 & 0 & 0 & 0 & 0 \\
\hline C1-Programas... & UU & VV & Ww & $\mathbf{x x}$ & YY & $\mathbf{Z Z}$ & AAA & BBB & $\operatorname{CCC} 5$ & DDD & EEE & FFF & GGG & $\mathrm{HHH}$ & III & JנJ & KKK & LLL & MMM & NNN & ÑÑÑ & 000 & PPP & \\
\hline Descripción & 0 & 0 & 0 & 0 & 0 & 0 & 0 & 0 & 0 & 0 & 1 & 0 & 0 & 0 & 0 & 0 & 0 & 0 & 0 & 1 & 0 & 0 & 0 & \\
\hline Logotipo & 0 & 0 & 0 & 0 & 0 & 0 & 0 & 0 & 0 & 0 & 0 & 0 & 0 & 0 & 0 & 0 & 0 & 0 & 0 & 1 & 0 & 0 & 0 & \\
\hline Comités, grupos & 0 & 0 & 0 & 0 & 0 & 0 & 0 & 0 & 0 & 0 & 0 & 0 & 0 & 0 & 0 & 0 & 0 & 0 & 0 & 0 & 0 & 0 & 0 & \\
\hline C2-P.Estratégicos & $\mathbf{A}$ & B & C & D & $\mathbf{E}$ & $\mathbf{F}$ & $\mathbf{G}$ & $\mathrm{H}$ & $I$ & $\mathrm{~J}$ & $\mathbf{K}$ & $\mathbf{L}$ & $M$ & $\mathbf{N}$ & $\tilde{\mathbf{N}}$ & 0 & $\mathbf{P}$ & $\mathbf{Q}$ & $\mathbf{R}$ & $S$ & $\mathbf{T}$ & U & $\mathbf{v}$ & $\mathbf{w}$ \\
\hline Líneas, ejes & 0 & 1 & 0 & 1 & 0 & 0 & 1 & 0 & 0 & 0 & 0 & 0 & 1 & 0 & 0 & 1 & 0 & 0 & 0 & 1 & 0 & 0 & 0 & 0 \\
\hline Objetivos & 1 & 1 & 0 & 1 & 0 & 0 & 1 & 1 & 1 & 0 & 0 & 0 & 0 & 1 & 0 & 0 & 0 & 0 & 0 & 0 & 0 & 0 & 0 & 0 \\
\hline Otros & 0 & 1 & 0 & 0 & 0 & 1 & 1 & 1 & 1 & 0 & 0 & 1 & 0 & 0 & 0 & 0 & 0 & 0 & 0 & 0 & 0 & 0 & 0 & 0 \\
\hline C2-P.Estratégicos & $\mathbf{x}$ & $\mathbf{Y}$ & $\mathbf{z}$ & AA & BB & CC & DD & EE & FF & GG & HH & II & J J & KK & LL & MM & NN & $\tilde{\mathbf{N} N}$ & 00 & PP & QQ & $\mathbf{R R}$ & SS & TT \\
\hline Líneas, ejes & 1 & 1 & 1 & 0 & 0 & 1 & 0 & 0 & 0 & 0 & 0 & 0 & 0 & 0 & 0 & 0 & 0 & 1 & 0 & 0 & 0 & 1 & 0 & 1 \\
\hline Objetivos & 0 & 0 & 0 & 0 & 0 & 1 & 1 & 0 & 0 & 0 & 0 & 0 & 0 & 0 & 0 & 0 & 0 & 1 & 0 & 0 & 0 & 1 & 0 & 1 \\
\hline Otros & 0 & 0 & 0 & 0 & 0 & 0 & 0 & 0 & 0 & 0 & 0 & 0 & 1 & 0 & 1 & 0 & 0 & 1 & 0 & 0 & 0 & 1 & 0 & 1 \\
\hline C2-P.Estratégicos & UU & VV & WW & $\mathbf{X X}$ & $\mathbf{Y Y}$ & $\mathbf{Z Z}$ & AAA & BBB & $\operatorname{CCC} \mathrm{L}$ & DDD & EEE & FFF & GGG & $\mathrm{HHH}$ & III & JJ & KKK & LLL & MMM & NNN & ÑÑÑ & 000 & PPP & \\
\hline Líneas, ejes & 0 & 0 & 0 & 0 & 0 & 0 & 0 & 0 & 0 & 1 & 0 & 0 & 1 & 0 & 0 & 0 & 1 & 0 & 0 & 0 & 0 & 1 & 1 & \\
\hline Objetivos & 0 & 0 & 0 & 0 & 0 & 0 & 0 & 0 & 0 & 0 & 1 & 0 & 1 & 0 & 0 & 0 & 0 & 0 & 0 & 0 & 0 & 0 & 0 & \\
\hline Otros & 0 & 0 & 0 & 0 & 1 & 0 & 0 & 0 & 0 & 0 & 0 & 0 & 0 & 0 & 0 & 1 & 0 & 0 & 0 & 0 & 0 & 1 & 1 & \\
\hline C3-Edificios & A & B & C & D & $\mathbf{E}$ & $\mathbf{F}$ & $\mathbf{G}$ & $\mathbf{H}$ & $\mathbf{I}$ & J & $\mathrm{K}$ & $\mathbf{L}$ & $M$ & $\mathbf{N}$ & $\tilde{\mathbf{N}}$ & 0 & $\mathbf{P}$ & $\mathbf{Q}$ & $\mathbf{R}$ & $S$ & $T$ & U & $\mathbf{v}$ & w \\
\hline Ahorro energético & 0 & 1 & 0 & 0 & 0 & 1 & 0 & 0 & 0 & 0 & 0 & 0 & 0 & 0 & 0 & 1 & 0 & 0 & 0 & 0 & 0 & 0 & 0 & 0 \\
\hline $\begin{array}{l}\text { Adaptación disca- } \\
\text { pacitados }\end{array}$ & 0 & 1 & 0 & 0 & 0 & 1 & 0 & 0 & 0 & 0 & 0 & 0 & 0 & 0 & 0 & 0 & 0 & 0 & 0 & 0 & 0 & 0 & 0 & 0 \\
\hline Residuos & 0 & 1 & 0 & 0 & 1 & 1 & 0 & 0 & 0 & 0 & 0 & 0 & 0 & 0 & 0 & 1 & 0 & 0 & 0 & 0 & 0 & 0 & 0 & 0 \\
\hline Panel difusión & 0 & 1 & 0 & 0 & 0 & 0 & 0 & 0 & 0 & 0 & 0 & 0 & 0 & 0 & 0 & 1 & 0 & 0 & 0 & 0 & 0 & 0 & 0 & 0 \\
\hline C3-Edificios & $x$ & $\mathbf{Y}$ & $\mathbf{Z}$ & AA & BB & CC & DD & EE & \begin{tabular}{|l|}
$\mathbf{F F}$ \\
\end{tabular} & GG & $\mathbf{H H}$ & II & JJ & KK & LL & MM & NN & ÑÑ & 00 & PP & $\mathbf{Q Q}$ & $\mathbf{R R}$ & SS & TT \\
\hline Ahorro energético & 0 & 0 & 0 & 0 & 0 & 0 & 0 & 0 & 0 & 0 & 0 & 0 & 0 & 0 & 0 & 0 & 1 & 0 & 0 & 0 & 0 & 0 & 0 & 0 \\
\hline $\begin{array}{l}\text { Adaptación disca- } \\
\text { pacitados }\end{array}$ & 0 & 0 & 0 & 0 & 0 & 0 & 1 & 0 & 0 & 0 & 0 & 0 & 0 & 0 & 1 & 0 & 0 & 0 & 0 & 0 & 0 & 0 & 0 & 0 \\
\hline Residuos & 0 & 0 & 0 & 0 & 0 & 0 & 0 & 0 & 0 & 0 & 0 & 0 & 0 & 0 & 0 & 0 & 1 & 0 & 0 & 0 & 0 & 0 & 0 & 0 \\
\hline Panel difusión & 0 & 0 & 0 & 0 & 0 & 0 & 0 & 0 & 0 & 0 & 0 & 0 & 0 & 0 & 0 & 0 & 0 & 0 & 0 & 0 & 0 & 0 & 0 & 0 \\
\hline C3-Edificios & UU & VV & WW & $\mathbf{x X}$ & YY & $\mathbf{Z Z}$ & AAA & BBB & CCC & DDD & EEE & FFF & GGG & $\mathrm{HHH}$ & III & JנJ & KKK & LLL & MMM & NNN & ÑÑÑ & 000 & PPP & \\
\hline Ahorro energético & 0 & 0 & 0 & 0 & 0 & 0 & 0 & 0 & 0 & 0 & 0 & 0 & 0 & 0 & 0 & 0 & 0 & 0 & 0 & 1 & 0 & 0 & 0 & \\
\hline $\begin{array}{l}\text { Adaptación disca- } \\
\text { pacitados }\end{array}$ & 0 & 0 & 0 & 0 & 0 & 0 & 0 & 0 & 0 & 0 & 0 & 0 & 0 & 0 & 0 & 0 & 1 & 0 & 0 & 0 & 0 & 0 & 0 & \\
\hline Residuos & 0 & 0 & 0 & 0 & 0 & 0 & 0 & 0 & 0 & 0 & 1 & 0 & 0 & 0 & 0 & 0 & 0 & 0 & 0 & 1 & 0 & 0 & 0 & \\
\hline Panel difusión & 0 & 0 & 0 & 0 & 0 & 0 & 0 & 0 & 0 & 0 & 0 & 0 & 0 & 0 & 0 & 0 & 0 & 0 & 0 & 0 & 0 & 0 & 0 & \\
\hline
\end{tabular}




\begin{tabular}{|c|c|c|c|c|c|c|c|c|c|c|c|c|c|c|c|c|c|c|c|c|c|c|c|c|}
\hline C4-Colecciones & A & B & C & D & $\mathbf{E}$ & $\mathbf{F}$ & G & H & $\mathbf{I}$ & $\mathrm{J}$ & $\mathbf{K}$ & $\mathbf{L}$ & $M$ & $\mathbf{N}$ & $\tilde{\mathbf{N}}$ & 0 & $\mathbf{P}$ & $\mathbf{Q}$ & $\mathbf{R}$ & $S$ & $\mathbf{T}$ & U & $\mathbf{v}$ & $\mathbf{W}$ \\
\hline \begin{tabular}{|l|}
$\begin{array}{l}\text { Consorcios y } \\
\text { licencias }\end{array}$ \\
\end{tabular} & 1 & 1 & 1 & 1 & 1 & 1 & 1 & 1 & 1 & 0 & 0 & 0 & 0 & 0 & 0 & 1 & 0 & 0 & 0 & 1 & 0 & 1 & 1 & 0 \\
\hline Open Access & 0 & 0 & 0 & 0 & 1 & 0 & 0 & 1 & 0 & 0 & 0 & 0 & 1 & 1 & 0 & 0 & 0 & 0 & 0 & 0 & 0 & 0 & 0 & 0 \\
\hline Repositorios & 0 & 1 & & 1 & 1 & 1 & 0 & 0 & 0 & 0 & 1 & 0 & 0 & . & 1 & 1 & 7 & 0 & . & 0 & 1 & 1 & 1 & 1 \\
\hline Adquisiciones & 0 & 0 & 0 & 0 & 0 & 1 & 0 & 1 & 1 & 0 & 0 & 0 & 0 & 1 & 1 & 0 & 1 & 0 & 0 & 0 & 0 & 0 & 0 & 0 \\
\hline $\begin{array}{l}\begin{array}{l}\text { Expurgo y dupli- } \\
\text { cados }\end{array} \\
\end{array}$ & 0 & 1 & 0 & 0 & 0 & 1 & 1 & 0 & 1 & 0 & 0 & 1 & 0 & 0 & 0 & 0 & 1 & 0 & 0 & 0 & 0 & 0 & 1 & 0 \\
\hline Control consumo & 0 & 1 & 0 & 0 & 1 & 1 & 0 & 0 & 0 & 0 & 0 & 0 & 0 & 0 & 0 & 0 & 0 & 0 & 0 & 0 & 0 & 0 & 0 & 0 \\
\hline Fomento lectura & 0 & 0 & 1 & 1 & 0 & 0 & 1 & 0 & 1 & 0 & 1 & 1 & 1 & 0 & 0 & 0 & 0 & 0 & 0 & 0 & 0 & 0 & 0 & 0 \\
\hline Redes colaboración & 0 & 0 & 0 & 0 & 1 & 0 & 0 & 0 & 0 & 0 & 0 & 0 & 0 & 0 & 0 & 1 & 0 & 0 & 0 & 0 & 0 & 0 & 0 & 0 \\
\hline C4-Colecciones & $\mathbf{x}$ & $\mathbf{Y}$ & $\mathbf{Z}$ & AA & BB & CC & DD & EE & $\mathbf{F F}$ & GG & HH & II & Jנ & KK & LL & MM & NN & ÑÑ & 00 & PP & QQ & RR & SS & TT \\
\hline \begin{tabular}{|l}
$\begin{array}{l}\text { Consorcios y } \\
\text { licencias }\end{array}$ \\
\end{tabular} & 1 & 1 & 1 & 1 & 1 & 1 & 1 & 1 & 1 & 1 & 1 & 0 & 1 & 0 & 1 & 0 & 1 & 1 & 0 & 0 & 0 & 1 & 1 & 0 \\
\hline Open Access & 0 & 1 & 0 & 1 & 1 & 1 & 0 & 0 & 1 & 0 & 0 & 0 & 0 & 0 & 0 & 0 & 0 & 0 & 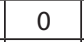 & 0 & 0 & 0 & 1 & 0 \\
\hline Repositorios & 1 & 1 & 1 & 0 & 1 & 1 & 1 & 1 & 0 & 1 & 1 & 0 & 0 & 0 & 0 & 0 & 1 & 1 & 0 & 0 & 0 & 1 & 1 & 1 \\
\hline Adqui & 1 & 0 & 0 & 0 & 0 & 0 & 1 & 0 & 0 & 0 & 0 & 0 & 0 & 0 & 0 & 0 & 1 & 1 & 0 & 0 & 0 & 0 & 0 & 0 \\
\hline $\begin{array}{l}\begin{array}{l}\text { Expurgo y dupli- } \\
\text { cados }\end{array} \\
\end{array}$ & 1 & 1 & 0 & 0 & 1 & 0 & 1 & 0 & 0 & 0 & 0 & 1 & 0 & 0 & 0 & 0 & 1 & 1 & 0 & 0 & 0 & 0 & 0 & 0 \\
\hline \begin{tabular}{|l|} 
Control consumo \\
\end{tabular} & 0 & 0 & 0 & 0 & 0 & 0 & 0 & 0 & 0 & 0 & 0 & 0 & 0 & 0 & 0 & 0 & 1 & 0 & 0 & 0 & 0 & 0 & 0 & 0 \\
\hline Fomento lectura & 0 & 0 & 0 & 0 & 0 & 0 & 1 & 0 & 0 & 0 & 0 & 0 & 0 & 0 & 0 & 0 & 1 & 0 & 0 & 0 & 0 & 1 & 1 & 0 \\
\hline Redes colaboración & 0 & 1 & 0 & 0 & 0 & 0 & 0 & 0 & 0 & 0 & 0 & 0 & 0 & 0 & 0 & 0 & 0 & 1 & 0 & 0 & 0 & 0 & 0 & 0 \\
\hline C4-Colecciones & UU & $\mathbf{~ V V}$ & WW & $x x$ & YY & $\mathbf{Z Z}$ & AAA & BBB & $\mathrm{CCC}$ & DDD & EEE & FFF & GGG & $\mathrm{HHH}$ & III & JנJ & KKK & LLL & MMM & NNN & ÑÑN & 000 & PPP & \\
\hline \begin{tabular}{|l}
$\begin{array}{l}\text { Consorcios y } \\
\text { licencias }\end{array}$ \\
\end{tabular} & 1 & 0 & 0 & 0 & 0 & 0 & 1 & 0 & 0 & 0 & 0 & 1 & 1 & 1 & 1 & 0 & 0 & 0 & 0 & 0 & 0 & 0 & 0 & \\
\hline Open access & 0 & 0 & 0 & 1 & 0 & 0 & 1 & 0 & 1 & 0 & 0 & 0 & 0 & 0 & 0 & 0 & 1 & 0 & 0 & 1 & 0 & 1 & 1 & \\
\hline Repos & 0 & 1 & 1 & 1 & 1 & 1 & 0 & 0 & 1 & 1 & 0 & 1 & 1 & 0 & 0 & 0 & 0 & 0 & 1 & 1 & 1 & 1 & 1 & \\
\hline Adquisiciones & 0 & 0 & 0 & 0 & 1 & 0 & 0 & 0 & 0 & 0 & 0 & 0 & 0 & 0 & 0 & 0 & 1 & 0 & 0 & 0 & 0 & 1 & 1 & \\
\hline \begin{tabular}{|l}
$\begin{array}{l}\text { Expurgo y } \\
\text { cados }\end{array}$ \\
\end{tabular} & 1 & 0 & 0 & 0 & 0 & 0 & 0 & 0 & 0 & 0 & 0 & 0 & 0 & 0 & 0 & 0 & 1 & 0 & 0 & 0 & 0 & 1 & 0 & \\
\hline Control consumo & 0 & 0 & 0 & 0 & 0 & 0 & 0 & 0 & 0 & 0 & 1 & 0 & 0 & 0 & 0 & 0 & 0 & 0 & 0 & 1 & 0 & 0 & 0 & \\
\hline Fomento lectura & 0 & 0 & 0 & 0 & 0 & 0 & 0 & 0 & 1 & 0 & 1 & 0 & 0 & 0 & 0 & 0 & 1 & 0 & 0 & 0 & 0 & 0 & 1 & \\
\hline Rede & 0 & 0 & 0 & 0 & 0 & 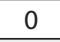 & 0 & 0 & 0 & 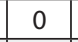 & 0 & 0 & 0 & 0 & 0 & 0 & 0 & 0 & 0 & 0 & 0 & 0 & 0 & \\
\hline $\begin{array}{l}\text { C5-Procesos y } \\
\text { servicios }\end{array}$ & A & B & C & D & E & $\mathbf{F}$ & G & H & I & J & K & $\mathbf{L}$ & $\mathbf{M}$ & $\mathbf{N}$ & $\tilde{\mathbf{N}}$ & 0 & $\mathbf{P}$ & $\mathbf{Q}$ & $\mathbf{R}$ & S & $\mathbf{T}$ & U & v & w \\
\hline $\begin{array}{l}\text { Servicios espe- } \\
\text { ciales }\end{array}$ & 0 & 1 & 0 & 1 & 0 & 0 & 1 & 1 & 1 & 0 & 0 & 0 & 1 & 0 & 1 & 1 & 0 & 0 & 0 & 0 & 0 & 0 & 0 & 0 \\
\hline $\begin{array}{l}\text { Cursos, campañas } \\
\text { o actividades }\end{array}$ & 0 & 0 & 0 & 0 & 1 & 0 & 0 & 0 & 0 & 0 & 0 & 0 & 0 & 0 & 0 & 1 & 0 & 0 & 0 & 0 & 0 & 0 & 0 & 0 \\
\hline \begin{tabular}{|l}
$\begin{array}{l}\text { Medidas de con- } \\
\text { cienciación }\end{array}$ \\
\end{tabular} & 0 & 1 & 0 & 0 & 1 & 0 & 0 & 0 & 0 & 0 & 0 & 0 & 0 & 0 & 0 & 1 & 0 & 0 & 0 & 0 & 0 & 0 & 0 & 0 \\
\hline $\begin{array}{l}\text { C5-Procesos y } \\
\text { servicios }\end{array}$ & 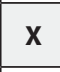 & $\mathbf{Y}$ & Z & AA & BB & CC & DD & EE & $\mathbf{F F}$ & GG & HH & II & J J & KK & LL & MM & $\mathbf{N N}$ & $\tilde{\mathbf{N}} \mathbf{N}$ & 00 & PP & QQ & RR & SS & TT \\
\hline $\begin{array}{l}\text { Servicios espe- } \\
\text { ciales }\end{array}$ & 1 & 0 & 0 & 0 & 0 & 0 & 1 & 1 & 0 & 0 & 0 & 0 & 0 & 0 & 0 & 0 & 0 & 1 & 0 & 0 & 0 & 1 & 0 & 0 \\
\hline $\begin{array}{l}\text { Cursos, campañas } \\
\text { o actividades }\end{array}$ & 0 & 0 & 0 & 0 & 0 & 0 & 0 & 0 & 0 & 0 & 0 & 0 & 0 & 0 & 0 & 0 & 0 & 0 & 0 & 0 & 0 & 0 & 0 & 0 \\
\hline $\begin{array}{l}\text { Medidas de con- } \\
\text { cienciación }\end{array}$ & 0 & 0 & 0 & 0 & 0 & 0 & 0 & 0 & 0 & 0 & 0 & 0 & 0 & 0 & 0 & 0 & 1 & 0 & 0 & 0 & 0 & 0 & 0 & 0 \\
\hline $\begin{array}{l}\text { C5-Procesos y } \\
\text { servicios }\end{array}$ & UU & Vv & ww & XX & YY & $\mathbf{Z Z}$ & AAA & BBB & CcC & DDD & EEE & FFF & GGG & HHH & III & ננر & KKK & LLL & MMM & NNN & ÑÑ & 000 & PPP & \\
\hline $\begin{array}{l}\text { Servicios espe- } \\
\text { ciales }\end{array}$ & 0 & 0 & 0 & 0 & 0 & 0 & 0 & 0 & 1 & 0 & 0 & 0 & 0 & 0 & 0 & 0 & 0 & 0 & 0 & 0 & 0 & 1 & 1 & \\
\hline $\begin{array}{l}\text { Cursos, campañas } \\
\text { o actividades }\end{array}$ & 0 & 0 & 0 & 0 & 0 & 0 & 0 & 0 & 0 & 0 & 0 & 0 & 0 & 0 & 0 & 0 & 0 & 0 & 0 & 0 & 0 & 0 & 0 & \\
\hline $\begin{array}{l}\text { Medidas de con- } \\
\text { cienciación }\end{array}$ & 0 & 0 & 0 & 0 & 0 & & 0 & & 0 & 0 & 1 & 0 & 0 & 0 & 0 & 0 & 0 & 0 & 0 & 1 & 0 & 0 & 0 & \\
\hline
\end{tabular}

Nota: Sí = 1, No = 0. Bibliotecas universitarias: A: Almería, B: Cádiz, C: Córdoba, D: Granada, E: Huelva, F: Jaén, G: Málaga, H: Pablo Olavide, I: Sevilla, J: Zaragoza, K: San Jorge, L: La Laguna, M: Las Palmas de Gran Canaria, N: Cantabria, Ñ. Castilla-La Mancha, O: Burgos, P: Católica de Ávila, Q: Europea Miguel de Cervantes, R: IE Universidad, S: León, T: Pontificia de Salamanca, U: Salamanca, V: Valladolid, W: Abat Oliva CEU, X: Autònoma de Barcelona, Y: Barcelona, Z: Girona, AA: Internacional de Catalunya, BB: Lleida, CC: Oberta de Catalunya, DD: Politècnica de Catalunya, EE: Pompeu Fabra, FF: Ramon Llull, GG: Rovira i Virgili, HH: Vic, II: Alfonso X El Sabio, JJ: Alcalá de Henares, KK: Antonio de Nebrija, LL: Autónoma de Madrid, MM: Camilo José Cela, NN: Carlos III de Madrid, ÑÑ: Complutense de Madrid, OO: Univ. a Distancia de Madrid, PP: Europea de Madrid, QQ: Francisco de Vitoria, RR: Nacional de Educación a Distancia (UNED), SS: Politécnica de Madrid, TT: U. Pontificia de Comillas, UU: Rey Juan Carlos, VV: San Pablo-CEU, WW: Navarra, XX: Pública de Navarra, YY: Alicante, ZZ: Cardenal Herrera CEU, AAA: Jaume I, BBB: Miguel Hernández, CCC: Politécnica de Valencia, DDD: Valencia, EEE: Extremadura, FFF: A Coruña, GGG: Santiago de Compostela, HHH: Vigo, III: Illes Balears, JJJ: Internacional de La Rioja, KKK: La Rioja, LLL: Deusto, MMM: País Vasco, NNN: Oviedo, ÑÑÑ: Católica de San Antonio, OOO: Murcia, PPP: Politécnica de Cartagena. 
Anexo 2. Repositorios

\begin{tabular}{|c|c|c|}
\hline Universidad & Repositorio & URL \\
\hline A Coruña & RUC. Repositorio Institucional de la Universidad de La Coruña & http://ruc.udc.es/dspace \\
\hline Alicante & RUA. Repositorio Institucional de la Universidad de Alicante & http://rua.ua.es/dspace \\
\hline $\begin{array}{l}\text { Autònoma de } \\
\text { Barcelona }\end{array}$ & DDD. Deposito Digital de Documentos de la UAB & http://ddd.uab.cat \\
\hline Barcelona & DD. Depósito Digital de la UB & http://diposit.ub.edu/dspace \\
\hline Burgos & Repositorio institucional UBU & $\begin{array}{l}\text { http://www.ubu.es/bubu/es/bub-biblioteca-digital/repositorio- } \\
\text { institucional-ubu-documentacion }\end{array}$ \\
\hline Cádiz & $\begin{array}{l}\text { Rodin. Repositorio de Objetos de Docencia e Investigación de la } \\
\text { Universidad de Cádiz }\end{array}$ & http://rodin.uca.es:8081/xm/ui \\
\hline Cantabria & UCrea. Repositorio Abierto de la Universidad de Cantabria & http://repositorio.unican.es/xmlui \\
\hline $\begin{array}{l}\text { Cardenal Herre- } \\
\text { raCEU }\end{array}$ & CEU. Repositorio Institucional & http://dspace.ceu.es \\
\hline Complutense & E-Prints Complutense & http://eprints.ucm.es \\
\hline Córdoba & Helvia. Repositorio Institucional de la Universidad de Córdoba & http://helvia.uco.es/xmlui \\
\hline $\begin{array}{l}\text { Carlos III de } \\
\text { Madrid }\end{array}$ & Repositorio E-Archivo & http://e-archivo.uc3m.es \\
\hline $\begin{array}{l}\text { Castilla La } \\
\text { Mancha }\end{array}$ & RUIdeRA. Repositorio Universitario Institucional de Recursos Abiertos & https://ruidera.uclm.es/xmlui \\
\hline $\begin{array}{l}\text { Católica de } \\
\text { Murcia }\end{array}$ & Repositorio Digital UCAM & http://repositorio.ucam.edu/jspui \\
\hline Girona & Dugidocs & http://dugi-doc.udg.edu \\
\hline Granada & Digibug. Repositorio institucional de la Universidad de Granada & http://digibug.ugr.es \\
\hline Huelva & Arias Montano. Repositorio Institucional de la Universidad de Huelva & http://rabida.uhu.es/dspace \\
\hline $\begin{array}{l}\text { Las Palmas de } \\
\text { Gran Canaria }\end{array}$ & Acceda. Documentación Científica de la Ulpgc en abierto & http://biblioteca.ulpgc.es/repositorioinstitucional \\
\hline Lleida & Repositorio Abierto UdL & http://repositori.udl.cat \\
\hline León & Bulería. Repositorio Institucional de la Universidad de León & https://buleria.unileon.es \\
\hline Murcia & Digitum. Repositorio institucional de la Universidad de Murcia & http://digitum.um.es/xmlui \\
\hline Navarra & Dadum. Depósito Académico Digital de la Universidad de Navarra & http://dspace.unav.es/dspace \\
\hline $\begin{array}{l}\text { Oberta de } \\
\text { Catalunya }\end{array}$ & Repositorio Institucional O2, la Oberta en abierto & http://openaccess.uoc.edu/webapps/o2 \\
\hline Oviedo & RUO. Repositorio Digital UniOVI & http://buo.uniovi.es/ebiblioteca/nuestrorepositorio \\
\hline País Vasco & ADDI. Archivo Digital Docencia Investigación & https://addi.ehu.es/?locale=es \\
\hline $\begin{array}{l}\text { Politécnica de } \\
\text { Cartagena }\end{array}$ & Repositorio digital UPCT & http://repositorio.bib.upct.es/dspace \\
\hline $\begin{array}{l}\text { Politècnica de } \\
\text { Catalunya }\end{array}$ & UPCommons. Portal de acceso abierto al conocimiento de la UPC & http://upcommons.upc.edu \\
\hline $\begin{array}{l}\text { Politécnica de } \\
\text { Madrid }\end{array}$ & Archivo Digital UPM & http://oa.upm.es \\
\hline $\begin{array}{l}\text { Politécnica de } \\
\text { Valencia }\end{array}$ & $\begin{array}{l}\text { RiuNet. Repositorio Institucional de la Universidad Politécnica de } \\
\text { Valencia }\end{array}$ & $\begin{array}{l}\text { http://www.upv.es/entidades/ABDC/infoweb/bg/ } \\
\text { info/798082normalc.html }\end{array}$ \\
\hline Pompeu Fabra & Repositorio Digital de la UPF & http://repositori.upf.edu \\
\hline $\begin{array}{l}\text { Pontificia de } \\
\text { Comillas }\end{array}$ & Repositorio Comillas & $\begin{array}{l}\text { http://biblioteca.upcomillas.es/opacrepositorio/abnetcl.exe/ } \\
\text { O7020/ID561674ec/NT1?ACC }=120 \& F O R M=1\end{array}$ \\
\hline $\begin{array}{l}\text { Pontificia de } \\
\text { Salamanca }\end{array}$ & Summa. Repositorio Documental UPSA & http://summa.upsa.es \\
\hline $\begin{array}{l}\text { Pública de } \\
\text { Navarra }\end{array}$ & Repositorio institucional Academica-e & http://academica-e.unavarra.es \\
\hline Rovira i Virgili & Repositorio Institucional & http://www.urv.cat/repositori_institucional/index.html \\
\hline Salamanca & Repositorio Documental Gredos & http://gredos.usal.es/jspui \\
\hline San Pablo Ceu & Repositorio institucional CEU & http://dspace.ceu.es \\
\hline $\begin{array}{l}\text { Santiago de } \\
\text { Compostela }\end{array}$ & Repositorio institucional USC & http://dspace.usc.es \\
\hline UNED & Repositorio institucional e-Spacio UNED & http://e-spacio.uned.es/fez \\
\hline Valencia & Roderic. Repositorio de Contenido Libre & http://roderic.uv.es \\
\hline Valladolid & UVaDoc. Repositorio Documental de la Universidad de Valladolid & http://uvadoc.uva.es \\
\hline Vic & RiuVic. Repositorio Institucional de la Universidad de Vic & http://repositori.uvic.cat \\
\hline Zaragoza & Zaguán. Repositorio Digital de la Universidad de Zaragoza & http://zaguan.unizar.es \\
\hline
\end{tabular}

